\title{
Examining the Impact of Advanced Digital Economy on Employment in China Under COVID-19
}

\author{
Tianyu $\mathrm{Cao}^{1, *}$
}

\author{
${ }^{1}$ Birmingham Business School, College of Social Science, University of Birmingham, Birmingham B15 2TT, United \\ Kingdom \\ *Corresponding author's Email: TXC974@student.bham.ac.uk
}

\begin{abstract}
This paper investigates the impact of advanced digital technology on employment in China under specific Covid-19 period. By reviewing and summarizing previous relevant papers, we have drawn an conclusion that the rapid digital progress in China demonstrates significant contribution to transformation of employment structure in China although it might cause potential technological unemployment risks. In response to the development of digital economy under global pandemic, we raise some effective suggestions for government on how to properly utilize digital technology to boost employment under current situation.
\end{abstract}

Keywords: Digital economy, Covid-19, Employment, Transformation

\section{INTRODUCTION}

Digital economy is an advanced economic stage after agricultural economy and industrial economy. Digital economy takes digital knowledge and information as the key production factors, digital technology as the core driving force, modern information network as an important carrier and the effective use of information and communication technology as an important driving force for efficiency improvement and economic structure optimization. Through the deep integration of digital technology and the real economy, it continuously improves the digitization, networking and intelligence level of economic society, accelerates the reconstruction of economic development and governance model of the new economic form. As an economic concept in our context, digital economy is an economic form in which human beings guide and realize the rapid optimal allocation and regeneration of resources through the identification, selection, filtering, storage and use of big data so as to achieve high-quality economic development. Digital economy, as a relatively broad concept, can be included in the category of economic forms that directly or indirectly use data to guide resources to play a role in promoting the development of productivity. At the technical level, it includes big data, cloud computing, Internet of things, blockchain, artificial intelligence, 5G communication and other emerging technologies. At the application level, the new retail e-commerce platform for daily life services represented by Alibaba Jingdong Meituan and the online education \& remote working platform such as Zoom or Tencent conference are all exemplary applications of digital economy in our real life. Due to the rapid development of digital economy in China, employment in almost all industries have been profoundly influenced. Each technological revolution has brought of profound adjustment in employment structure. The new economic form not only replaces part of the traditional jobs but also creates new jobs. Since the number of new employment opportunities created is greater than the replaced employment, it is a result that the total amount of employment is increasing and the employment structure is upgrading ${ }^{[1]}$. Digital economy is a new economic form after agricultural economy and industrial economy, which has a profound impact on the current employment structure. China is accelerating the digital transformation progress, which has a huge impact on the employment situation. First, digital economy has reconstructed the employment mode. Second, digital economy has promoted the flexible employment in China to achieve four major changes: from marginal supplement to important components, from a small number of fields to diversified fields, from low-level employment to high-level employment, and from 
passive choice to active participation. Third, digital economy has promoted the optimization and upgrading of employment. Despite the fact that automation contributes to boosting labour productivity, it is far from sufficient to create more employment opportunities since each additional robot used in manufacturing will replace 3.3 workers $^{[2]}$. Hit by automation, the employed population of the US has decreased by $0.18 \%-0.34 \%$ as well as due to slow creation of new jobs. Based on previous studies it can be concluded that low-skilled labors with relatively low level education attainment are most vulnerable to substitution of automation progress. Due to the outbreak of COVID-19 global pandemic since 2020, low and middle-skilled workers are being faced with fueling threat of displacement and becoming obsolete faster as the global pandemic accelerates digital transformation. Due to the shortage of labour supply caused by pandemic induced mobility restrictions, the use of automation in companies will sharply increase to get rid of reliance upon workers and the automation process of some jobs is expected to be accelerated and persist as pandemic continues.

The structural unemployment risk that digital economy may bring about mainly include three causes in summary. First, machine \& robots replacement may cause structural unemployment risk. Second, the replacement of new and old business forms also causes structural unemployment risks. Third, transformation and upgrading of industrial structure causes structural unemployment risk. Despite the potential structural unemployment risk caused by digital economy, under the Covid-19 global pandemic since 2020, the benefit of digital economy on saving employment has been greatly augmented. Macro data showed that the epidemic has formed a huge pressure on the performance of China's employment market. From January to may 2020, China would create 4.6 million new urban jobs, a year-on-year decrease of 1.37 million. China's urban unemployment rate will remain between $5.0 \%$ and $5.3 \%$ in each month of 2019. In January 2020, the urban unemployment rate rose by $0.2 \%$ year-on-year. Since February, it has risen sharply to $6.2 \%$ with a year-on-year increase of $0.9 \%$ and it has remained at a high level of about $6.0 \%$ for several consecutive months. Nevertheless, online catering and food delivery service has been promoted by both digital technology and pandemic outbreak and it thus would compensate for employment loss due to the lockdown policy during pandemic. Specifically, delivery rider has become a major job position to absorb employment during the epidemic period. Learned from "Elemo", the platform has attracted hundreds of thousands of registered riders since COVID-19 outbreak. The field of digital economy has become an important channel to absorb employment and promote consumption. According to the data released by Meituan, the delivery platform of Meituan recruited 75000 new labor force to become delivery riders from
January 20th to February 23rd in 2020. According to the Meituan rider employment report in 2019 and 2020 released by Meituan Research Institute ${ }^{[3]}$, the total number of riders earning income through the platform will reach 3.987 million in 2019. After the outbreak, Meituan platform has recruited 336000 new riders from January 20th to March 18th, indicating that digital platform has contributed vastly to the employment absorption and creation under pandemic.

In summary, previous studies focusing on employment under digital economy progress and Covid19 pandemic almost reach an agreement that despite the fact that the pandemic has detrimental impact on a number of traditional industries, digital economy such as online food delivery and shared employee platform which is driven by both digital technology transformation and pandemic lockdown play a major role in creating employment opportunities and compensating for employment loss since it has provided temporary employment opportunities for those who lost their original jobs due to pandemic and for those who could not find other suitable jobs. In this study, we have summarized the contributing impact of advanced digital technology on employment as well as structural unemployment risks it may bring and examined compensatory effect of digital economy on employment loss due to global pandemic. It is thus valuable to further explore the detailed effect of digital economy development on different aspects of employment in China especially under global pandemic background.

\section{HOW THE DEVELOPMENT OF DIGITAL ECONOMY IMPACTS EMPLOYMENT IN CHINA UNDER COVID-19 PANDEMIC?}

\subsection{Technological unemployment risks}

Technical unemployment is a type of unemployment caused by technological progress. In the process of economic growth, it is an inevitable trend of technological progress that capital and technology intensive technology are more and more widely used in production and more and more advanced equipment replace the labor of workers. In this way, the relative reduction of labor demand will increase unemployment. In addition, in the process of economic growth, the decline in the relative price of capital goods and the rise in the relative price of labor also aggravate the trend of machinery replacing workers which also aggravates the unemployment. The progress and wide application of digital technology not only expand the scale of employment, optimize the employment structure and promote flexible employment, but also bring a certain technological unemployment risk which has aroused worldwide attention. With the further development of Internet plus, intelligent manufacturing and new 
generation of artificial intelligence, the technological unemployment caused by digital technology will further spread in manufacturing and service industries, which will pose threat to the employment promotion at present and in the future. First, in the service sector, the "Internet plus" push forward has made some jobs "overloaded" and "outdated" replaced. For example, the development of e-commerce has accelerated the decline of the traditional commodity trading market, resulting in that from 2007 to 2016, the proportion of employment in the commodity trading market of more than 100 million yuan in the total wholesale and retail employment has decreased from $33 \%$ to less than $19 \%$, with a relative reduction of more than 1.5 million jobs. In the field of manufacturing, with the continuous rise of human costs and the in-depth promotion of intelligent manufacturing, manufacturing enterprises in coastal areas have joined the wave of automation upgrading. For example, Shenzhen rapoo company has replaced $75 \%$ of its employees with robots, and Foxconn has invested tens of thousands of robots Every year in recent years, with a total staff reduction of more than 60000 . But on the whole, the scale of the current technological unemployment is small and it is quickly absorbed by the new jobs created in the service industry, thus the unemployment risk could be effectively resolved. Second, the short-term pressure will be concentrated in the low-end manufacturing employment market. In the next three to five years, there might be a round of technological unemployment in the manufacturing sector. There are two main reasons: one is that there is a vast deficiency in machine replacement. The labor rate of China's manufacturing industry is still lower than that of the United States and other developed countries. Although the number of robots used is increasing rapidly, the total ownership is low. The density of industrial robots is only $1 / 8$ of that of South Korea, 1/6 of that of Japan, 1/5 of that of Germany, and 1/3 of that of the United States; the other is that the level and structure of manufacturing jobs in China is low, and the potential risk of being replaced is high. China's manufacturing industry is mainly at the middle and low end of the global value chain, mainly engaged in routine work such as production and assembly. Employees are only required to have lower skills, which is more likely to be replaced by machines. Statistics shows that in 2016 , more than $75 \%$ of the employees with high school and below education level accounted for more than 100 million in China's manufacturing industry. To deal with the short-term technological unemployment risk, we need to strengthen entrepreneurship and innovation and resolve the impact by driving employment transfer through new kinetic energy. Third, the unemployment risk caused by human-computer integration has become the focus in the medium and long term. In the next 10 or 20 years, the biggest challenge lies in the substitution of artificial intelligence and other technologies for service work. Since China is transforming into a service society, service industry is the main position of new employment and the best absorber of technological unemployment in manufacturing industry. If the service jobs are widely replaced by machines, it will not only affect this part of the replaced service jobs, but also destroy the employment transfer mechanism between the manufacturing industry and the service industry, and bring double pressure to employment promotion. Moreover, the employment structure and quality of China's service industry are relatively low, which has obvious potential substitution risk. Although from the perspective of previous waves of technological unemployment, the total amount of employment has increased instead of decreasing and the long-term employment situation is optimistic, the unemployment caused by human-computer integration in the service industry is still a problem that needs to be valued and solved in the future.

\subsection{Flexible employment}

The digital economy progress especially under Covid-19 global pandemic has greatly facililated the transformation of employment structure mainly by enhancing groundbreaking form of flexible employment. Flexible employment relationships in general will take another, maybe even more recognized position in the labor market ${ }^{[4]}$. "Shared employee", for example, is an exemplary application of flexible employment. Under the joint promotion of digital economy and exogenous epidemic factors, sharing employment platform flourished during Covid19. Specifically, the booming delivery industry has successfully created large number of employment opportunities especially for those who have lost their original jobs due to pandemic. Delivery industry increased the employment of the residents in Hubei vastly. From the source of new riders, $53.9 \%$ of the new riders were registered residence in Hubei province. Affected by the epidemic situation, the local employment rate of new riders in Hubei province has increased, ranking from 15th in 2019 to 5th during the epidemic period, which indicates the situation that a large number of riders in Hubei province were employed nearby and earned income for their lives. Besides, the delivery industry has absorbed a large number of employees from the secondary and primary industry in which jobs are mainly based on routinely physical labour and very less likely to allow remote working. The shutdown caused by the epidemic was the main reason for them to join the riders' team. It can be concluded that delivery industry has enhanced the elasticity of employment of riders and maintained the stability of social employment during pandemic since the booming food delivery industry has greatly covered the employment loss of labour mainly from jobs that are most vulnerable to the devastating effect of the pandemic as these jobs usually require low education 
level and hardly allow employees to work remotely such that these workers are most inclined to be unemployed during lockdown period. Delivery industry has vastly satisfied the employment demand of workers from these aforementioned jobs and greatly alleviated unemployment. Besides, according to China's shared economy development report $2020^{[5]}$, under the situation of limited offline activities caused by shutdown measurement, the areas of live short video or knowledge sharing have grown rapidly and the demand for employment in these areas accordingly increased sharply. According to the data, within one month after returning to work in the Spring Festival in 2020, the number of live broadcast related part-time jobs increased by $166.09 \%$, more than twice the growth rate of full-time jobs. In the first half of 2020, Baidu Wenku opened more than 40000 knowledge stores, directly promoting the employment of nearly 1 million part-time or full-time content creators. From August 2019 to August 2020, 20 million 970 thousand people earned income from creative work, live broadcasting Tiktok and electricity providers. In the field of life services, the total number of riders who obtained income through Meituan platform was 2.952 million in the first half of 2020, an increase of $16.4 \%$ year on year. On the one hand, new jobs relying on the shared platform involve a wide range of fields and demonstrate strong inclusiveness. There are not only knowledge intensive and complex labor posts suitable for college students such as creative planning, software design, online education but also skilled labor posts suitable for relatively low education level groups such as takeaway riders, online car hailing driver, cloud customer service etc, It creates broader space and more opportunities for the employment of key social groups. The survey shows that about $20.4 \%$ of the full-time drivers on Didi platform are engaged in online car hailing due to laidoff, unemployment and other reasons, $41.1 \%$ are from manufacturing industry, $13.6 \%$ are from transportation industry, and $4.9 \%$ are from steel, coal and other decapacity industries. In the first half of 2020, the number of new riders on Meituan platform who are from the poverty-stricken households and have set up files and registered cards are nearly 80000; On the other hand, platform enterprises can timely adjust the supply of labor force according to the changes of market supply and demand to promote the cross industry flow of labor force and reduce frictional unemployment. During the epidemic period, the riders of Meituan platform absorbed a large number of employees from the secondary and tertiary industries. $35.2 \%$ of the riders came from factory workers, $31.4 \%$ from entrepreneurs or small businesses themselves, and $17.8 \%$ from office staff. With the rapid development of new forms of employment, new jobs such as online distributor, ecommerce anchor, online consultant are constantly emerging. In 2019 and 2020, China has released three batches of 38 new occupations more than half of which are related to platform economy, such as digital manager, internet of things installation and debugging worker, UAV driver and e-athlete. For example, in the field of service industry, with the continuous advancement of digital transformation, there are many distinctive new types of work across more than 200 life service segments. The emergence of new jobs vividly reflects the changes of "quantity" and "quality" of economic development which not only enriches the types of employment but also promotes the changes of the employment structure of the whole society.

\subsection{Youth employment}

It is more likely for youth ages 16 to 24 to be unemployed than the adult population from traditional perspective ${ }^{[6]}$. Over the past two decades, it has been observed that youth have been two to three times more likely to be unemployed than working-age adults. Economic downturns hit youth particularly hard. After the Great Recession of 2007-2008, youth unemployment increased more rapidly than the overall level of unemployment. In 2010, the peak of the economic downturn, youth unemployment climbed to $18.4 \%$. Unemployment was particularly high among younger youth (those ages 16 to 19). More than a quarter were unemployed in 2010. Since 2010, unemployment declined steadily for all age groups, reaching a low in 2019. However, COVID-19 pandemic caused a stark shift in youth unemployment in early 2020 as lockdown measurement was implemented, unemployment began to rise again. With the adoption of social distancing measures, unemployment rates across all age groups increased starting in April 2020. Employment data from the first two months into the pandemic indicate that the youth unemployment rate is above 25 percent. In April 2020, more than a quarter of youth were unemployed: 31.9 percent of youth ages 16 to 19 and 25.7 percent of youth ages 20 to 24 . In May, the unemployment rate decreased slightly, but a quarter of youth ages 16 to 24 were still unemployed. The COVID-19 pandemic imposes several challenges that might exacerbate youth economic vulnerability in the coming years. Even during positive business cycles, youth are economically more vulnerable because they tend to work in seasonal, casual, temporary, or part-time jobs, often without paid leave and benefits and most jobs that youth do cannot be done remotely. Nevertheless, the digital transformation has obvious compensatory effect on youth employment that suffered largely from the current economic downturn induced by global pandemic. Since the data of age distribution of Meituan takeaway riders in 2018 shows that riders ages between 25 and 40 take up more than $83 \%$ of overall investigated riders, it indicates that youth riders make up the vast majority of this industry. Thus, youth is the major beneficiary of digital employment platform such as Meituan takeaway platform which has created and 
absorbed employment vastly as we mentioned above. Besides, according to the age distribution of Kwaishou and Tiktok users in 2019, nearly 95\% users are under the age of 40 and over $83 \%$ users are under 30 . It obviously indicates that youth play a leading role in utilizing short video platform and thus could realize flexible employment during pandemic. In summary, the disadvantage of youth on employment exacerbated by the pandemic can be alleviated to a large extent by the flexible employment empowered by the digital economy progress.

\section{SUGGESTIONS ON POLICIES TO IMPROVE EMPLOYMENT THROUGH DIGITAL TECHNOLOGY UNDER PANDEMIC}

\subsection{Promote the digital transformation of the real economy}

During the lockdown period, the traditional business model of the real economy was greatly restricted which brought great challenges to employment. The development of online business mode contributes to maintaining employment stability by promoting the integration of real economy and the Internet to accelerate digital transformation. Taking the education industry and catering industry as an example, the traditional face-to-face in-person teaching mode and restaurant eating for here business mode were severely devastated by the pandemic. However, demand of people for education and food consumption did not decline significantly. In order to meet people's demand for education and food consumption in the special period of lockdown, the online education platform and food delivery platform empowered by digital economy thus obtain precious development opportunities. Therefore, the booming online education industry plays a crucial role in maintaining employment stability of teachers in China during the epidemic period by protecting most of them from being unemployed. As we mentioned earlier, food delivery platform has contributed vastly to maintaining employment stability during pandemic since its large absorbing effect on employment has created huge amounts of jobs for the unemployed from other industries. Based on the investigation questionnaire made by Meituan 2020, nearly $30 \%$ of catering businesses turned to delivery in the short term in response to the impact of the epidemic. In the case of a serious decline in dine-in, many catering businesses realized that the combination of multiple business models would better cope with the risks and try to invest more resources in takeout in the short term. In early February 2020, a telephone survey of 33000 catering businesses organized by Meituan Research Institute showed that nearly $30 \%$ of the interviewees decided to gradually turn to takeaway business half of whom were investing more resources in takeaway business and changing their business focus. In the short term, takeout has become an important way for catering businesses to tide over the epidemic crisis. In the long run, the epidemic has accelerated the online process of the catering industry to a certain extent and the combination of online and offline will become the new normal of the development of catering industry in China. With the digital restructuring of the catering industry, online delivery service is facing new development opportunities and greatly boosts employment. Other industries of real economy can learn experience from online education and delivery service platform to carry out digital transformation by utilizing the integration with the Internet and develop new business mode of joint online and offline. Moreover, the digital transformation of real economy enterprises should not only be a temporary emergency measure responding to the pandemic, but should also be incorporated into the long-term development plan of enterprises and become a normalized business model.

\subsection{Government support and guidance for medium and small corporations under pandemic}

According to Notice of the Ministry of industry and information on coping with the outbreak of Covid-19 pandemic and helping SMEs resume production and tide over difficulties released on February 9th 2020, the government intended to support enterprises mainly in two aspects, finance and innovation. In order to further strengthen the financial support for small and mediumsized enterprises, first of all, government will promote the implementation of the national financial and tax support policies for key enterprises in epidemic prevention and assist local small and medium-sized enterprises included in the central list of key enterprises for epidemic control to apply for discount support and tax preference according to policies and regulations. The competent departments of small and medium-sized enterprises in Hubei, Zhejiang, Guangdong, Henan, Hunan, Anhui, Chongqing, Jiangxi, Beijing, Shanghai and other provinces shall strengthen policy implementation and services for small and mediumsized enterprises included in the list of key enterprises for epidemic prevention and control in the region. Local governments should give further support on the basis of discount interest on loans from the central government. Second, encourage local governments to introduce relevant financial support policies. Give full play to the role of special funds for the development of small and medium-sized enterprises at the same level and set up special relief funds where conditions permit to increase support for small and medium-sized enterprises seriously affected by the epidemic. We should encourage all localities to take into account the actual situation of local small and medium-sized enterprises 
affected by the epidemic, reduce taxes and administrative fees in accordance with the law and promote the introduction of financial support policies such as reducing property rents, delaying or appropriately returning social insurance premiums, delaying tax payment, reducing the cost of production factors, and increasing subsidies for skills training and job stabilization for enterprise employees to effectively reduce the cost burden of small and medium-sized enterprises. When it comes to innovation, in order to support enterprise digital transformation. The government will vigorously promote Internet platform services for small and medium-sized enterprises and actively promote online office, video conference, remote cooperation and digital management so as to comprehensively improve the management informatization level of small and medium-sized enterprises to help enterprises providing offline services innovate business models and expand online services. Government will accelerate the deployment of 5G and industrial Internet applications, promote a number of industrial software applications suitable for small and medium-sized enterprises and support small and medium-sized enterprises to enhance their agile manufacturing and lean production capacity. Government will support the small and medium-sized enterprises in the industrial cluster to make up for the lack of resources and capabilities of a single enterprise through network cooperation, integrate the decentralized manufacturing capabilities through collaborative manufacturing platform and realize the sharing of technology, production capacity and orders. In addition, the government should support enterprises to improve the level of intelligent manufacturing to guide large enterprises and professional service institutions to launch cloud manufacturing platform and cloud service platform for small and medium-sized enterprises, as well as develop products, solutions and toolkits suitable for the needs of small and medium-sized enterprises intelligent manufacturing. Government will also promote the cloud deployment of small and mediumsized enterprises business systems, dock with the industrial Internet platform, guide the basic and qualified small and medium-sized enterprises to speed up the intelligent transformation of production lines and promote the deployment and application of low-cost and modular intelligent manufacturing equipment and systems in small and medium-sized enterprises.

\subsection{Support and encourage workers to participate in online vocational skills training}

To effectively implement online training schemes, the following suggestions should be followed. First, open online vocational skills training resources free of charge. During the epidemic period, relying on the online vocational skills training platform, key courses for workers should be made free of charge. Government will increase the supply of vocational skills training course resources covering major industries and types of work, actively guide and encourage large enterprises, colleges and universities, vocational colleges (including technical colleges) and social training institutions to open online vocational skills training resources free of charge during the epidemic period. Second, improve the quality of online vocational skills training resources by making full use of the portal, mobile app, wechat applet and other channels to improve the accessibility of online vocational skills training. Optimize the registration process and user interface of online vocational skills training and improve the convenience of online vocational skills training. Make full use of mobile Internet technology to promote the extension of skills training services to mobile intelligent terminals and selfservice terminals. Third, improve online vocational skills training supporting services. Relying on the online vocational skills training platform, we will carry out activities such as exhibition of great country craftsmen and winners of the world skills competition during the epidemic period, so as to improve the attractiveness of online vocational skills training. Basic telecommunication enterprises will give preference to online vocational skills training during the epidemic period. By means of information technology, the whole process tracking management of learning situation and training effect can be realized and the evaluation after training can be completed well.

\section{CONCLUSION AND DISCUSSION}

This paper analyses the influence of advanced digital economy on employment in China under the particular pandemic period, based on various research findings obtained by other related papers. We have found that the impact of digitalization on employment mainly comprises higher technological unemployment risk that a large number of low skilled workers in traditional manufacturing industry are highly vulnerable to automation replacement, more flexible employment forms that overturn the traditional employment relationship and change employment structure, bringing especially young people with unprecedented more job opportunities under Covid-19. Faced with this extraordinary situation, through investigations and analysis of relevant articles, we come up with some suggestions on government policies to improve employment by using digital technology during pandemic period, including promoting the digital transformation of traditional real economy enterprises, government financial support for small and medium companies and enhancing online vocational skills of workers, to cope with current employment difficulties induced by global pandemic.

However, there are still some limitations in our paper. For example, we only examined the digital 
economy progress and employment in China. However, considering the situation varies greatly across countries, the impact of digitalization on employment may differ greatly in different countries. To improve this limitation, for future research we could further conduct investigations on other countries with different developing levels and then compare the results horizontally to observe more distinct effect of digitalization progress on employment. Besides, more advanced methodology could be used to conduct empirical analysis.

\section{REFERENCES}

[1]China Academy of Information and Communications Technology (2018). Report of China's digital economy development and employment.

[2]Asian Development Bank(2020).COVID-19, Technology, and Polarizing Jobs. No. 147.

[3]Meituan Research Institute(2019). Meituan rider employment report.

[4]Spurk, D. , \& Straub, C. (2020). Flexible employment relationships and careers in times of the covid-19 pandemic. Journal of Vocational Behavior, 119.

[5]National information center(2020). China's shared economy development report.

[6]Inanc, Hande. "Breaking down the numbers: What does COVID-19 mean for youth unemployment." Mathematica Policy Research (2020): 1-22 